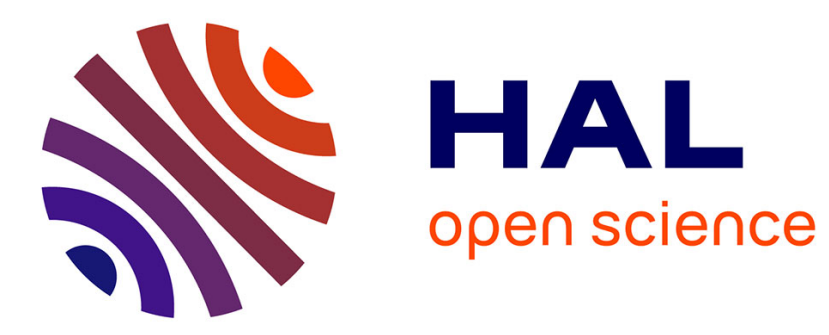

\title{
The invisible niche: Weakly density-dependent mortality and the coexistence of species
}

\author{
Thilo Gross, Andrew M. Edwards, Ulrike Feudel
}

\section{To cite this version:}

Thilo Gross, Andrew M. Edwards, Ulrike Feudel. The invisible niche: Weakly density-dependent mortality and the coexistence of species. Journal of Theoretical Biology, 2009, 258 (1), pp.148. 10.1016/j.jtbi.2009.01.018 . hal-00554565

\section{HAL Id: hal-00554565 \\ https://hal.science/hal-00554565}

Submitted on 11 Jan 2011

HAL is a multi-disciplinary open access archive for the deposit and dissemination of scientific research documents, whether they are published or not. The documents may come from teaching and research institutions in France or abroad, or from public or private research centers.
L'archive ouverte pluridisciplinaire $\mathbf{H A L}$, est destinée au dépôt et à la diffusion de documents scientifiques de niveau recherche, publiés ou non, émanant des établissements d'enseignement et de recherche français ou étrangers, des laboratoires publics ou privés. 


\section{Author's Accepted Manuscript}

The invisible niche: Weakly density-dependent mortality and the coexistence of species

Thilo Gross, Andrew M. Edwards, Ulrike Feudel

PII:

S0022-5193(09)00032-0

DOI: doi:10.1016/j.jtbi.2009.01.018

Reference: YJTBI 5443

To appear in: $\quad$ Journal of Theoretical Biology

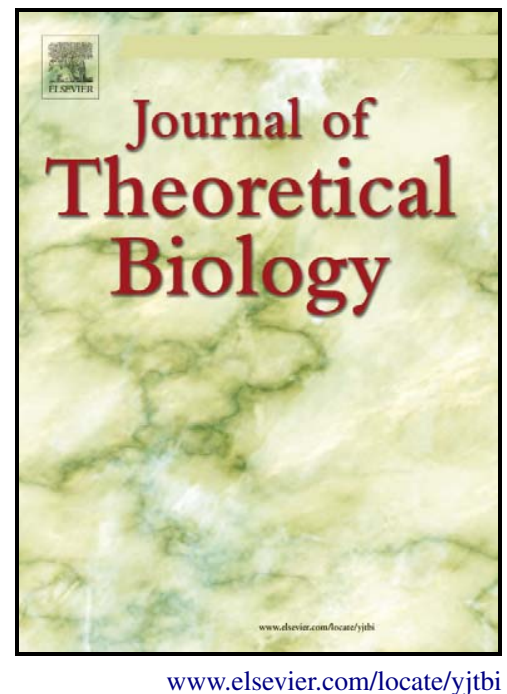

Received date: $\quad 21$ November 2008

Revised date: $\quad 22$ January 2009

Accepted date: $\quad 22$ January 2009

Cite this article as: Thilo Gross, Andrew M. Edwards and Ulrike Feudel, The invisible niche: Weakly density-dependent mortality and the coexistence of species, Journal of Theoretical Biology (2009), doi:10.1016/j.jtbi.2009.01.018

This is a PDF file of an unedited manuscript that has been accepted for publication. As a service to our customers we are providing this early version of the manuscript. The manuscript will undergo copyediting, typesetting, and review of the resulting galley proof before it is published in its final citable form. Please note that during the production process errors may be discovered which could affect the content, and all legal disclaimers that apply to the journal pertain. 


\title{
The invisible niche: weakly density-dependent mortality and the coexistence of species
}

\author{
Thilo Gross * \\ Max-Planck Institute for Physics of Complex Systems, Nöthnitzer Straße 38, \\ 01187 Dresden, Germany \\ Andrew M. Edwards \\ Pacific Biological Station, Fisheries and Oceans Canada, 3190 Hammond Bay \\ Road, Nanaimo, British Columbia, V9T 6N7, Canada
}

Ulrike Feudel

Institut für Chemie und Biologie des Meeres, Carl von Ossietzky Universität,PF 2503, 26111 Oldenburg, Germany.

\begin{abstract}
Weakly density-dependent effects, characterized by fractional scaling exponents close to one, are rarely studied in the ecological literature. Here, we consider the effect of an additional weakly density-dependent term on a simple competition model. Our investigation reveals that weak density-dependence opens up an "invisible niche". This niche does not constitute a new mechanism for coexistence, but is a previously unexplored consequence of known mechanisms. In the invisible niche a weaker competitor can survive at very low density. Coexistence thus requires large habitat size. Such niches, if found in nature, would have a direct impact on species-area laws and species-abundance curves and should therefore receive more attention.
\end{abstract}

* Corresponding author.

Email: thilo.gross@physics.org 


\section{Introduction}

Density dependence is ubiquitous in ecological interactions. However, in models it is generally only taken into account if it is sufficiently strong to be directly evident in experiments. This is particularly obvious in the mathematical treatment of mortality. Out of convention, mortality terms in models are often taken to be linear plus an optional quadratic term. Other possible choices, such as terms with non-integer exponents, are only rarely used (Steele and Henderson, 1992; Caswell and Neubert, 1998; Edwards and Yool, 2000; Edwards and Bees, 2001). By contrast, results from physics suggest that in complex systems fractional exponents are the rule rather than the exception (Mandelbrot, 1967; Bak, 1996; Wilson, 1975)). In this paper, we argue that weakly density-dependent mortality, characterized by fractional exponents slightly greater than one, could potentially have a strong qualitative impact on ecological dynamics; their investigation may shed new light on important current questions such as species-abundance curves and species-area laws. We illustrate this point by investigating the effect of weakly densitydependent mortality terms on one of the oldest and most fundamental ecological concepts: the competitive exclusion principle.

The competitive exclusion principle states that the number of populations that can coexist indefinitely cannot exceed the number of ecological niches in the system (Hardin, 1960). In modern literature this insight is often attributed to Gause (1934), who provided evidence from laboratory experiments. However, 
when Gause stated the principle it already "had been expressed often in the naturalist literature and was felt to be obvious and therefore unremarkable" (Kingsland, 1988, p. 146). Indeed, the competitive exclusion principle had been addressed by Nägeli (1874) and Grinnell $(1904,1917)$ and was treated mathematically by Haldane (1924) and Volterra (1926) and also, in a different way, already by Nägeli (1874).

In order to comprehend the way in which the concept of competitive exclusion has shaped the thinking of ecologists even before Gause's time, consider Darwin's theory of evolution. Although the focus of the theory is on individuals rather than populations the notion of survival of the fittest, coined by Herbert Spencer, already implies the exclusion of the others. This dependence of Darwinian evolution on competitive exclusion illustrates the role of the competitive exclusion principle as a conceptual cornerstone of ecological theory.

It is interesting to note that Gause's work was often cited in the 1930s to advocate the need for more laboratory experiments, while the ecological implications for real world ecosystems were hardly ever discussed (Kingsland, 1988, p. 162-3). Kingsland (1988, p. 158) offers the explanation that the competitive exclusion principle was perceived as a "laboratory curiosity" with little relevance to natural systems. Only in response to Gause's restatement of the principle in 1939 and subsequent works by Lack (1947) and Hutchinson (1947) it was realized that the competitive exclusion principle may hold the key to the struc- 
ture of ecological communities. This insight inspired further work by Slobodkin (1961), Odum (1961), McArthur and Levins (1964), Rescigno and Richardson (1965), and others who extended the mathematical models on which the competitive exclusion principle is based.

A more detailed investigation of the competitive exclusion principle revealed that the number of species is not necessarily limited by the number of limiting resources in the system (as Gause had claimed), but by the number of "niches". However, stating that the number of populations that can persist in a system cannot exceed the number of niches is tautological, since a niche is generally defined as some parameter space in which persistence is possible (Slobodkin, 1961; Levin, 1970). It has been pointed out by Hutchinson (1961) and Slobodkin (1961) that - despite its tautological nature - the competitive exclusion principle is nevertheless useful if it is understood as a working guideline to investigate the cases in which it apparently fails. Indeed, in many real world ecosystems the stable coexistence of very similar species seems to be the rule (Hutchinson, 1959). Perhaps the most well known examples are Lack's work on ground finches (Lack, 1947), MacArthur's study of warblers (MacArthur, 1958) and Hutchinson's paradox of the plankton (Hutchinson, 1961).

The investigation of examples of species coexistence has revealed a number of ways in which competitive exclusion can be avoided. These include selective predation (Holt and Lawton, 1994; Vandermeer and Maruca, 1998; 
Chase et al., 2002), the presence of parasitoids (Hastings and Godfray, 1999), certain types of social behavior (Hutchinson, 1947) which enable stationary coexistence, as well as several mechanisms that allow coexistence in nonstationary states, such as self-organized population dynamics (Koch, 1974b; Armstrong and McGehee, 1976b,a; McGehee and Armstrong, 1977; Armstrong and McGehee, 1980; Huisman and Weissing, 1999), external forcing (Koch, 1974a; Ebenhöh, 1994; Abrams, 2004) and spatio-temporal dynamics (Solé et al., 1992; Bracco et al., 2000; Chesson, 2000; Roy et al., 2004; Wilson and Abrams, 2005).

The works cited above have provided many answers to the question of why coexistence of similar species is possible despite the competitive exclusion principle. In this article we do not propose a new mechanism but rather focus on a different question: Why are there so many different factors that enable the coexistence in nature, while exclusion seems to be almost unavoidable in laboratory experiments? One answer to this question is that many experiments by design exclude some factors that can enable coexistence. Here we show that another, less obvious, answer may exist. Weakly density-dependent effects may enable coexistence depending on the system size; consequently coexistence becomes possible in large natural systems, but is impossible in small laboratory-sized systems.

We start in Sec. 1 by restating some previous results on coexistence in a more general way. In Sec. 2 we study density-dependent mortality in a realistic general model. These investigations reveal apparently discontinuous behavior in 
the limit of weakly density-dependent mortality. These findings are then illustrated in a specific example in Sec. 3. The same example system is used in Sec. 4 to investigate the nature of the discontinuity in more detail. In particular we show that coexistence through weakly density-dependent mortality is strongly dependent on the system size. Finally, in Sec. 5, we discuss the potential role of weakly density-dependent effects in nature.

\section{Competitive exclusion in mathematical models}

In this section we review some well known results on the competitive exclusion principle from a different perspective. In particular we focus on the by-now canonical reasoning for competitive exclusion in density-independent models and the way it is avoided in density-dependent models. While this provides only few new insights it restates the known results with greater generality, illustrates important concepts, and sets the stage for the new results presented in the subsequent sections.

\subsection{Competitive exclusion in density-independent models}

A typical setup in which competitive exclusion is studied consists of two populations of consumers who compete for a single limiting resource. We denote the density of the consumers by $X$ and $Y$, respectively and the density of the resource by $R$. In a simple conceptual model the dynamics of the consumers 
can be described by the ordinary differential equations

$$
\begin{aligned}
& \dot{X}=g_{\mathrm{x}}(R) X-m_{\mathrm{x}} X, \\
& \dot{Y}=g_{\mathrm{y}}(R) Y-m_{\mathrm{y}} Y,
\end{aligned}
$$

where $g_{\mathrm{x}}$ and $g_{\mathrm{y}}$ denote the functional response of the consumer populations and $m_{\mathrm{x}}$ and $m_{\mathrm{y}}$ denote their mortality rates. In addition to the two equations given above, there has to be at least one additional algebraic or differential equation that governs the dynamics of the resource. However, for the discussion presented in this paper, this additional equation is only of secondary importance and will not be stated explicitly. The reasoning presented here is therefore valid for biotic as well as abiotic resources.

While the conceptual model in Eq. (1) provides only a very abstract picture of natural systems, it describes several paradigmatic models discussed in ecological literature. For example it reduces to Volterra's model for competition for an abiotic resource $\left(g_{\mathrm{x}}(R)=c_{\mathrm{x}} R, g_{\mathrm{y}}(R)=c_{\mathrm{y}} R, R=R_{\max }-F(X, Y)\right.$, with positive constants $c_{\mathrm{x}}, c_{\mathrm{y}}$ and $R_{\max }$ and a monotonically increasing response function $F(X, Y)$ ) (Volterra, 1926). Or, Armstrong and McGehee's example for coexistence on a single biotic resource $\left(g_{\mathrm{x}}(R)=\eta_{\mathrm{x}} c_{\mathrm{x}} R, g_{\mathrm{y}}(R)=\eta_{\mathrm{y}} c_{\mathrm{y}} R /\left(R_{\mathrm{s}}+R\right)\right.$, $\left.\mathrm{d} R / \mathrm{dt}=r R(1-R / K)-X g_{\mathrm{x}}(R) / \eta_{\mathrm{x}}-Y g_{\mathrm{y}}(R) / \eta_{\mathrm{y}}\right)($ Armstrong and McGehee, 1976b).

For the special cases mentioned above, it is well known that competitive exclusion generally prevents the stationary coexistence of the consumer populations (Levin, 1970). Following a widely accepted line of reasoning, we can show the 
same for our general model. Setting the time derivatives of both population densities to zero, yields the conditions

$$
\begin{aligned}
& 0=g_{\mathrm{x}}\left(R^{*}\right) X^{*}-m_{\mathrm{x}} X^{*}, \\
& 0=g_{\mathrm{y}}\left(R^{*}\right) Y^{*}-m_{\mathrm{y}} Y^{*},
\end{aligned}
$$

where the asterisks indicate densities in the stationary state. Since we are interested in solutions with strictly positive densities, we can divide the conditions by $X^{*}$ and $Y^{*}$, respectively. In the two resulting equations

$$
\begin{aligned}
& 0=g_{\mathrm{x}}\left(R^{*}\right)-m_{\mathrm{x}}, \\
& 0=g_{\mathrm{y}}\left(R^{*}\right)-m_{\mathrm{y}}
\end{aligned}
$$

the resource density $R^{*}$ is the only remaining variable. We are thus, faced with a system of two equations that depend only on one variable. Such a system is over determined and hence can not be solved except in certain degenerate cases. The chances of finding such a degenerate case in nature are of measure zero, unless another mechanism, such as, say, evolution, favors the degeneracy. In the case of competitive exclusions no such mechanism has been described. Hence, it is safe to restrict the investigation to the generic cases. In the following we focus therefore only on the structurally stable solutions. Since a structurally stable solution to Eq. (3) does not exist, coexistence is generically impossible.

While the reasoning presented above is certainly elegant, it contains a subtle flaw: the conclusion was based on the fact that an over determined system has no structurally stable solutions. However, the condition stated in Eq. (2) was 
not over determined. In general it is not possible to transform a system that is not over determined into an over determined one. In the example above the overdeterminedness arose since we were able to eliminate two variables completely from the system, which in turn was only possible because of the assumption that the growth and the mortality terms of the populations depend on the population density in exactly the same way, e.g., the exact density independence of both mortality and growth rates in the consumer population. The critical dependence of the reasoning on the exact linearity of the functions shows that the model on which the conclusions are based is in itself structurally unstable.

To make this clear: on the one hand we have argued that in general only structurally stable solutions should play a role in nature. On the other hand we have focused on a model which is in itself structurally unstable, and for the very same reason may describe real world systems incorrectly. This problem does not arise from the fairly general way of treatment presented here, but is inherent in many proofs of the competitive exclusion principle, which apply a similar reasoning.

\subsection{Coexistence in simple density-dependent models}

As we have already mentioned it can be justified to focus on a structurally unstable situation, if there is some mechanism that favors that specific situation. This will be discussed in more detail below. For the moment let us assume that 
there is no biological requirement that demands exactly density-independent growth and mortality terms.

In nature many mechanisms are observed that give rise to density dependence, these mechanisms include overcrowding, diseases, predator interference, prey defense, social interactions, parasites, degradation of resource quality, and the consumer's dependence on other additional resources. Note that these mechanisms generally give rise to a less-than-linearly increasing growth rates and/or more-than-linearly increasing mortality rates. It appears therefore highly unlikely that in any species both the growth and the mortality term exhibit exactly the same dependence on population density.

For the sake of simplicity we keep the growth rate in this paper densityindependent and focus on the case of density-dependent mortality rates. While the case of density-dependent growth rates is mathematically equivalent and therefore completely analogous, the underlying mechanisms are less clear from an ecological point of view.

If density-dependent effects are taken into account, the structural instability in the model vanishes and stationary coexistence becomes possible. This can be illustrated in the following system:

$$
\begin{aligned}
& \dot{X}=g_{\mathrm{x}}(R) X-m_{\mathrm{x}} X^{p_{\mathrm{x}}}, \\
& \dot{Y}=g_{\mathrm{y}}(R) Y-m_{\mathrm{y}} Y^{p_{\mathrm{y}}} .
\end{aligned}
$$

In contrast to the model from the previous section we have added the exponents $p_{\mathrm{x}}>1$ and $p_{\mathrm{y}}>1$ on the mortality terms to reflect the density 
dependence. In analogy to Eq. (2) we obtain the conditions

$$
\begin{aligned}
& 0=g_{\mathrm{x}}\left(R^{*}\right) X^{*}-m_{\mathrm{x}} X^{* p_{\mathrm{x}}} \\
& 0=g_{\mathrm{y}}\left(R^{*}\right) Y^{*}-m_{\mathrm{y}} Y^{* p_{\mathrm{y}}}
\end{aligned}
$$

Solving for the population densities reveals the feasible steady state

$$
\begin{aligned}
& X^{*}=\sqrt[p_{\mathrm{x}}-1]{g_{\mathrm{x}}\left(R^{*}\right) / m_{\mathrm{x}}}, \\
& Y^{*}=\sqrt[p_{\mathrm{y}}-1]{g_{\mathrm{y}}\left(R^{*}\right) / m_{\mathrm{y}}} .
\end{aligned}
$$

Further investigations show that this steady state is in general dynamically stable in a large parameter space (Gross, 2004).

This model seems to imply that unconditional coexistence is possible. However, the per-capita mortality rates vanish for small population densities. Hence, the observed coexistence may be artificial and 'unbiological'. While we will study a more realistic model in the following, note that competitive exclusion only occurs in the degenerate density-independent case $\left(p_{X}=p_{Y}=1\right)$, while generic models, even with arbitrarily weak density dependence $\left(p_{x}, p_{y}\right.$ close to 1), predict stationary coexistence.

\section{Density dependence in a realistic model}

In the previous section we have shown that the canonical proof of competitive exclusion is based on a structurally unstable model. Furthermore, we have shown that when the structural instability is removed, coexistence is generically possible. Let us now investigate the coexistence of competing populations 
in a more realistic model

$$
\begin{aligned}
& \dot{X}=g(R) X-m_{\mathrm{x}} X^{p_{\mathrm{x}}}-d_{\mathrm{x}} X, \\
& \dot{Y}=g(R) Y-m_{\mathrm{y}} Y^{p_{\mathrm{y}}}-d_{\mathrm{y}} Y,
\end{aligned}
$$

where we have for the sake of simplicity assumed that both competitors exhibit the same functional response $g(R)$. In addition to the density-dependent mortality terms we have included density-independent mortality terms which correspond to per-capita losses of $d_{\mathrm{x}}$ and $d_{\mathrm{y}}$, respectively. These terms model basic metabolic costs and natural old-age mortality; they ensure that the percapita mortality can not drop below a certain minimum value.

\subsection{Conditions for stable coexistence}

In our model, it is apparent that one or both populations become extinct if $d_{\mathrm{x}}$ and/or $d_{\mathrm{y}}$ are chosen in such a way that they surpass the growth rate in the steady state. Unconditional coexistence can therefore not be expected. This notion can be confirmed by computation of the non-trivial steady state, which reveals

$$
\begin{aligned}
& X^{*}=\sqrt[p_{\mathrm{X}}-1]{\frac{g\left(R^{*}\right)-d_{\mathrm{x}}}{m_{\mathrm{X}}}}, \\
& Y^{*}=\sqrt[p_{\mathrm{y}}-1]{\frac{g\left(R^{*}\right)-d_{\mathrm{y}}}{m_{\mathrm{Y}}}} .
\end{aligned}
$$

Positive solutions for Eq. (8) exist if $g\left(R^{*}\right) \geq d_{\mathrm{x}}, g\left(R^{*}\right) \geq d_{\mathrm{y}}$.

We can imagine three possible stationary outcomes: a) extinction: both competitors go extinct; b) competitive exclusion: the stronger competitor survives, but lowers the equilibrium resource level $R^{*}$ so far that the weaker competitor 
becomes extinct; c) coexistence: the competitors approach an equilibrium in which their per-capita mortality rates match up $g\left(R^{*}\right)=m_{\mathrm{x}} X^{* p_{\mathrm{x}}-1}+d_{\mathrm{x}}=$ $m_{\mathrm{y}} Y^{* p_{\mathrm{y}}-1}+d_{\mathrm{y}}$ and long-term coexistence is possible.

In this paper our focus is on cases b) and c). Let us assume that there are sufficient resources in the system to permit the survival of at least one of the populations. In order to determine whether coexistence is possible we ask whether the populations can invade in a system, in which the other is already established.

Let us first assume that population $\mathrm{X}$ is the stronger competitor. We denote the stationary density of $\mathrm{X}$ in the absence of $\mathrm{Y}$ by $X_{+}$and the corresponding resource density by $R_{\mathrm{x}}$. In order for $Y$ to invade, the per-capita growth rate of $\mathrm{Y}$ at an arbitrarily low density $Y_{0}$ has to be positive. This yields the invasibility condition

$$
g\left(R_{\mathrm{x}}\right)-m_{\mathrm{x}} X_{+}^{p_{\mathrm{x}}-1}-d_{\mathrm{x}}=0<g\left(R_{\mathrm{x}}\right)-m_{\mathrm{y}} Y_{0}^{p_{\mathrm{y}}-1}-d_{\mathrm{y}}
$$

and therefore

$$
m_{\mathrm{x}} X_{+}^{p_{\mathrm{x}}-1}-m_{\mathrm{y}} Y_{0}^{p_{\mathrm{y}}-1}>d_{\mathrm{y}}-d_{\mathrm{x}}
$$

By considering the case where $Y$ is the resident $X$ is the invader we find the analogous condition

$$
m_{\mathrm{x}} X_{0}{ }^{p_{\mathrm{x}}-1}-m_{\mathrm{y}} Y_{+}{ }^{p_{\mathrm{y}}-1}<d_{\mathrm{y}}-d_{\mathrm{x}}
$$


Combining Eq. (10) and Eq. (11) we obtain

$$
m_{\mathrm{x}} X_{0}^{p_{\mathrm{x}}-1}-m_{\mathrm{y}} Y_{+}^{p_{\mathrm{y}}-1}<\delta<m_{\mathrm{x}} X_{+}^{p_{\mathrm{x}}-1}-m_{\mathrm{y}} Y_{0}^{p_{\mathrm{y}}-1}
$$

where

$$
\delta:=d_{\mathrm{y}}-d_{\mathrm{x}}
$$

denotes the difference in the density-independent mortality rates. For $p_{\mathrm{x}}>1$ and $p_{\mathrm{y}}>1$ the density-dependent mortality terms at arbitrarily small densities $X_{0}$ and $Y_{0}$ can be ignored. This yields the simplified condition

$$
-m_{\mathrm{y}} Y_{+}{ }^{p_{\mathrm{y}}-1}<\delta<m_{\mathrm{x}} X_{+}^{p_{\mathrm{x}}-1}
$$

Since the left hand side of this inequality is negative while the right hand side is positive, there is always a finite interval for $\delta$ in which each of the species can invade a system in which the other species is present. Mutual invasibility implies that there is a stable attractor on which the population densities are positive. Consequently the coexistence of similar species, which differ only moderately in their density-independent mortality is generically possible. Since the attractor is not necessarily a steady state, one could argue that this may be just another example of the well-known coexistence on a limit cycle Armstrong and McGehee (1980). Note however, that coexistence via the Armstrong-McGehee mechanism is impossible for predators with identical functional responses. Based on the analogy to the simpler model investigated in the previous section, it is therefore reasonable to assume that 
the stable attractor in the model is - at least in a large parameter space - the steady state described by Eq. (8).

\subsection{The limit of weak density dependence}

It is well known that strong density dependence can enable the coexistence of populations. However, among similar populations strong density-dependent effects which affect the populations separately are relatively rare. However, weak density dependence can arise in numerous ways, since no ecological interaction is likely to be exactly linear. In the following we investigate the impact of weakly density-dependent functions on coexistence in the framework proposed above.

In order to simplify notation let us assume that the density-dependent mortality terms are identical for both competitors. This assumption is not critical, since it will become clear in the following that for the questions considered here only the density-dependent mortality term of the stronger competitor is of importance. Furthermore, let us assume that the magnitude and exponent of the density-dependent mortality are identical for both populations, that is $m=m_{\mathrm{x}}=m_{\mathrm{y}}$ and $p=p_{\mathrm{x}}=p_{\mathrm{y}}$. We can write the mutual invasibility condition, Eq. (14) as

$$
-m\left(Y_{+}\right)^{p-1}<\delta<m\left(X_{+}\right)^{p-1}
$$

In the model proposed above, the density dependence can be weak in two 
different ways: First, the magnitude $m$ of the nonlinear mortality term can be small compared to that of the density-independent term $(m \ll d)$; we call this weak density-dependent mortality. Second, the exponent of the nonlinear term can be small, so that the density dependence in the nonlinear term is weak ( $p=1+\epsilon$ with $0<\epsilon \ll 1)$; this is called weakly density-dependent mortality.

The limit of weak density-dependent mortality corresponds to Eq. (15) for $m \rightarrow 0$. As we decrease $m$, the non-competitive densities $X_{+}$and $Y_{+}$increase, since lower density-dependent mortality generally entails higher equilibrium densities. However, if the system under consideration is a well-posed ecological model, $X_{+}$and $Y_{+}$converge to a finite limiting value for $m \rightarrow 0$. Therefore Eq. (15) shows that the permissible range for $\delta$ shrinks linearly as we approach $m=0$. In the limit $m \rightarrow 0$ structurally stable coexistence becomes impossible. This is shown in the left panel of Fig. 1.

In the following our focus is on the case of weakly density-dependent mortality. As we take the limit $p \rightarrow 1$ the densities $X_{+}$and $Y_{+}$can change. Note however, that we have assumed that the stronger competitor can survive in the system if the weaker competitor is absent. This implies $X_{+}>0$ and $Y_{+}>0$. Apart from pathological cases we can expect that the stronger competitor reaches a significant finite density even for $p=1$. We can therefore straightforwardly compute the limit of Eq. (15), which yields

$$
-m<\delta<m
$$


Note that even in the limit $p \rightarrow 1$ structurally stable coexistence is possible in a finite interval for $\delta$. However, based on the results of the previous section it is apparent that structurally stable coexistence is impossible for $p=1$. This apparent contradiction is resolved if we take the density of the weaker competitor into account. As we approach the density-independent limit the equilibrium density of the weaker competitor decreases. In the limit $p \rightarrow 1$ the density of the weaker competitor approaches zero. Therefore the transition from the weakly density-dependent to the density-independent case is still smooth in a certain sense. This point will become clearer in the example considered in the next section.

\section{An illustrative Example}

Let us consider the specific example system described by the equations

$$
\begin{gathered}
\dot{R}=\left(c_{\mathrm{in}}-R\right) r-G_{\mathrm{x}}(R) X-G_{\mathrm{y}}(R) Y, \\
\dot{X}=\eta_{\mathrm{x}} G_{\mathrm{x}}(R) X-m_{\mathrm{x}} X^{p}-d_{\mathrm{x}} X, \\
\dot{Y}=\eta_{\mathrm{y}} G_{\mathrm{y}}(R) Y-m_{\mathrm{y}} Y^{p}-d_{\mathrm{y}} Y,
\end{gathered}
$$

where $G_{\mathrm{x}}(R)=A_{\mathrm{x}} R /\left(k_{\mathrm{x}}+R\right)$ and $G_{\mathrm{y}}(R)=A_{\mathrm{y}} R /\left(k_{\mathrm{y}}+R\right)$. This system of equations describes the exploitative competition between two predators with Holling Type-II functional response for an abiotic nutrient, e.g., in a chemostat. The parameters $c_{\text {in }}$ and $r$ denote the concentration of nutrients in the inflow and the turnover rate respectively, while $\eta_{x}$ and $\eta_{y}$ denote the efficiency of biomass conversion. 
In the following we focus on the case $r=3, c_{\mathrm{in}}=20, \eta_{\mathrm{x}}=\eta_{\mathrm{y}}=1, m_{\mathrm{x}}=$ $m_{\mathrm{y}}=0.2, A_{\mathrm{x}}=A_{\mathrm{y}}=2, k_{\mathrm{x}}=4.5, k_{y}=5, d_{\mathrm{y}}=1$ and $d_{\mathrm{x}}=d$. Note that we have chosen the half saturation constants of the uptake kinetics in such a way that population $\mathrm{X}$ has a slight advantage. This was done in order to demonstrate that the results of the previous section do not depend critically on the assumption of identical uptake kinetics.

In order to compute the stationary solutions we set the left hand sides of Eqs. (17) to zero. In addition we divide the second and third line by the respective population density in order to remove the trivial solutions from the system. This yields

$$
\begin{gathered}
0=\left(c_{\mathrm{in}}-R\right) r-G_{\mathrm{x}}(R) X-G_{\mathrm{y}}(R) Y \\
0=G_{\mathrm{x}}(R)-m_{\mathrm{x}} X^{p-1}-d \\
0=G_{\mathrm{y}}(R)-m_{\mathrm{y}} Y^{p-1}-1
\end{gathered}
$$

We can now set the density of the weaker competitor to zero and solve the remaining system of two equations to compute the minimum value of $p, p_{\text {crit,x }}$, that is necessary for coexistence. For the case in which $\mathrm{X}$ is the weaker competitor we find

$$
p_{\text {crit }, \mathrm{x}}=\frac{\log Z_{0, \mathrm{x}}+\log Z_{1, \mathrm{x}}}{\log Z_{0, \mathrm{x}}},
$$

where

$$
\begin{gathered}
Z_{0, \mathrm{x}}=\frac{r}{2} \frac{\left(c_{\mathrm{in}}(d-2)+k_{\mathrm{x}} d\right)\left(k_{\mathrm{y}}(d-2)+k_{\mathrm{x}} d\right)}{(d-2) d k_{\mathrm{x}}} \\
Z_{1, \mathrm{x}}=\frac{1}{m_{\mathrm{y}}} \frac{k_{\mathrm{y}}(d-2)+k_{\mathrm{x}} d}{k_{\mathrm{x}} d-k_{\mathrm{y}}(d-2)}
\end{gathered}
$$


For the analogous case in which $\mathrm{Y}$ is the weaker competitor we obtain

$$
p_{\text {crit,y }}=\frac{\log Z_{0, \mathrm{y}}+\log Z_{1, \mathrm{y}}}{\log Z_{0, \mathrm{y}}}
$$

where

$$
\begin{aligned}
& Z_{0, \mathrm{y}}=\frac{r}{2} \frac{\left(c_{\mathrm{in}}-k_{\mathrm{x}}\right)\left(k_{\mathrm{y}}+k_{\mathrm{x}}\right)}{k_{\mathrm{x}}} \\
& Z_{1, \mathrm{y}}=-\frac{1}{m_{\mathrm{x}}} \frac{k_{\mathrm{y}}(d-2)+k_{\mathrm{x}} d}{k_{\mathrm{x}}+k_{\mathrm{y}}}
\end{aligned}
$$

The stability boundaries described by Eqs. $(19,21)$ are plotted in Fig. 2. The figure confirms our expectations based on the more general results shown in Fig. 1. In particular in the limit $p \rightarrow 1$ there is a finite range for $d$ in which coexistence is technically possible. The size of this range is approximately $2 m=m_{\mathrm{x}}+m_{\mathrm{y}}$ as can be expected from Eq. (16). Note however that the range is not centered around $d=1(\delta=0)$ since the uptake kinetics were chosen differently for the two competitors. The asymmetry of the plot in Fig. 2 for larger $p$ arises from the presence of a limiting value for $d$ beyond which the density-independent mortality rate of the weaker predator exceeds the maximum growth rate.

\section{Coexistence in systems of finite size}

The results presented above show that a finite coexistence region exists in models in which the density dependence is arbitrarily weak $\left(p_{x}, p_{y}\right.$ close to 1$)$, but is absent in density-independent models. The enigma presented by this apparent discontinuity is resolved if we consider coexistence in finite systems. 
So far we have implicitly assumed that positive equilibrium densities - no matter how small - are sufficient for coexistence. However, in finite systems the discreteness of individuals and demographic stochasticity introduce finite cut-off densities below which stochastic effects lead to rapid exclusion of one of the species.

In the following we take the finite size of the system into account and demand that the weaker competitor exceeds a given threshold density. In reality this density depends on the physical size of the habitat as compared to the individuals. Below we derive a formula that relates the permissible disadvantage of the weaker species $\delta$ to the threshold density that is required. We focus particularly on the threshold density of $10^{-3}$ and $10^{-30}$. The rationale for choosing these is the following: Compared to the density of the stronger competitor which is roughly of the order of one the density of $10^{-30}$ is so small that persistence is certainly impossible. Even if the stronger competitor were one of the most common microbial species and the system considered is of the size of the earth then the population of the weaker competitor consisted only of a few cells at best and rapidly went extinct because of demographic stochasticity. By contrast a density that is $10^{-3}$ that of the stronger competitor is still rare but certainly detectable if the competitor is a common species.

Let us return to the model introduced in the previous section and compute the parameter range in which both species exceed a given threshold density. This question is slightly more complicated than the one considered before, 
since straight forward computation of the corresponding conditions leads to a polynomial of fifth order. Also, naive numerical solutions are not feasible since terms of strongly different orders of magnitude arise and the computation of weakly linear exponentials is prone to give rise to numerical artifacts. We avoid these difficulties by deriving an implicit condition using the symbolic algebra software Maple.

Starting from Eq. (18) we first compute the value of $R^{*}$ that is consistent with a given set of population densities $X^{*}, Y^{*}$ by solving the first line for $R^{*}$. Note, that for fixed $X^{*}, Y^{*}$ the corresponding $R^{*}$ is independent of $d$ and $p$. For $R=R^{*}$ we can now compute the consistent value of $p=p^{*}$ that is required to obtain the chosen $X^{*}, Y^{*}$ by solving the third line of Eq. (18) for $p$. The result of this computation is independent of $d$. Finally we find the consistent value of $d=d^{*}$ by substituting $R^{*}$ and $p^{*}$ into the second line of Eq. (18) and solving for $d$.

The procedure described above provides us with analytical functions that map a given stationary state $X^{*}, Y^{*}$ to the corresponding parameter values $p^{*}\left(X^{*}, Y^{*}\right), d^{*}\left(X^{*}, Y^{*}\right)$. The computation of these values is technically simple, involving only factorization of polynomials of degrees one and two. Nevertheless the resulting functions are relatively lengthy and are therefore omitted here. For details please consult the maple worksheet that can be downloaded from the authors web page (Gross, 2007).

In the functions $p^{*}\left(X^{*}, Y^{*}\right), d^{*}\left(X^{*}, Y^{*}\right)$ we set the density of the weaker com- 
petitor, say $\mathrm{X}$, to a small value $q$ and vary the density of the stronger competitor over a reasonable range. The resulting line in parameter space bounds the parameter region in which the stationary density of $\mathrm{X}$ exceeds $q$. Repeating this procedure for both species and for different values of $q$ we obtain the diagram shown in Fig. 3.

Figure 3 shows that the transition at $p=1$ is continuous in a certain sense. The density of the weaker predator smoothly approaches zero as the exponent of closure is lowered. Thus, in finite systems in which demographic stochasticity introduces a density threshold, the permissible range of $d$ for which coexistence is possible smoothly approaches zero as $p \rightarrow 1$. Nevertheless note that relatively weak nonlinearities such as $p=1.05$ still allow for coexistence in a substantial range of $d$, albeit at low densities of the weaker competitor.

The central message we can get from the analysis of the model proposed here is the following: In systems with strictly density-independent mortality and growth the system size does not have a strong impact on the coexistence of species. In these systems coexistence at any finite density is generically impossible. Likewise, the size of the system is not important in systems with strongly density-dependent mortality. Here a competitor that is able to survive generally does so at a substantial density, and is therefore unlikely to be excluded because of demographic cut-offs. However, in the case of weakly density-dependent mortality there is a large parameter range in which coexistence is possible at very low densities. Therefore the size of the effective pa- 
rameter range in which coexistence is possible depends strongly on the relative size of the system (Fig. 4). For instance a planktonic species which manages to persist at, say $10^{-5}$ times the density of a stronger competitor can still reach high population numbers in an ocean, but would comprise of only a few individuals at most in a laboratory experiment. It could thus persist in the ocean, but would suffer demographic extinction in the laboratory.

\section{Discussion: Weakly density-dependent mortality in nature}

In this paper we have argued that weakly density-dependent effects in ecological interactions could open up an invisible niche: A population persisting in this niche is likely to have a small abundance and therefore could easily escape attention. Moreover, because of the niche's dependence on system size, coexistence in the invisible niche is probably not reproducible in laboratory-sized experiments. Finally, the weak nonlinearities that open up the niche are very difficult to measure by direct observation.

Despite the absence of direct evidence, invisible niches, if they exist, could play an important role in nature and thus may be detectable indirectly via their consequences. Coexistence induced by weakly density-dependent effects could play an important role in the maintenance of biodiversity. In particular in large ecosystems invisible niches might enable the persistence of many species at very low population density and might thus offer an answer to the enigma posed by species-abundance curves. In smaller systems the weaker competitors 
would be excluded. This not only offers an answer to our initial question, why coexistence seems to be the rule in nature while exclusion is commonly observed in the laboratory, but suggests a direct impact on species-area laws. It is conceivable that weakly density-dependent effects are thus detectable by their signature in species-area and species-abundance relationships. However, to determine this signature more theoretical work is certainly necessary.

In past models, linear and quadratic mortality terms have been used in part for simplicity and in part out of convention. Furthermore, it has been argued that the linear and quadratic mortality can be regarded as the first terms of a Taylor expansion of more complex functions. Note however, that weakly densitydependent functions are not Taylor expandable around the origin. Their effect can therefore not be captured by any combination of linear and quadratic terms.

In order to explore the impact of weakly density-dependent effects on speciesabundance and species-area laws the present results have to be extended to multiple species. For many of the arguments used here, this extension should be relatively straight forward. Recall that the competitors interact by lowering the level of the shared resource. But, in the presence of a strong competitor, the impact of weak competitors on the resource level will be minor as weakly density-dependent effects limit them to persist at very low density. The derived coexistence condition should therefore be approximately valid for multiple weak competitors independently, enabling the coexistence of an arbitrary 
number of populations.

Note that our model has every population suffering from a separate densitydependent mortality term. As every one of these terms constitutes a limiting factor, we would observe the coexistence of an arbitrary number of species only with the same number of limiting factors, which is hardly surprising. We should therefore ask whether it is necessary for coexistence to have a separate density-dependent mortality term for each of the weak competitors. On the one hand we were able to ignore the weakly density-dependent terms for the weaker competitor entirely in our derivation of the mutual invasibility conditions. On the other hand, one could argue that if such terms were neglected, the weak competitors themselves formed a system in which the classical competitiveexclusion principle applies. While this latter point is certainly valid, one could suspect that the presence of a strong competitor could fix the resource level very rigidly and thereby effectively decouple weaker competitors. However, more investigations in this directions are certainly necessary before anything can be said with certainty.

Although an empirical proof for the existence of weakly density-dependent effects will most likely be found in an indirect way, a direct search for weakly density-dependent processes would also be desirable. To our knowledge no such attempt has ever been undertaken. Although the functional form and in particular the scaling behavior at small densities is difficult to measure directly, the attempt to do so would at least reveal upper bounds for the strength of 
weakly density-dependent effects for the species under consideration.

Let us discuss the mechanisms by which weakly density-dependent effects could arise. While simple mechanistic models in general do not lead to fractional exponents, it known that scaling laws with such exponents often emerge in complex systems. In ecology such systems exist at least on two levels. First, fractional exponents could arise from complex internal processes in the individuals. For instance predators preying on the competitors will be more experienced in capturing the common competitor than the rare one. While learning can sometimes be described by sigmoid functional responses, the underlying mechanisms are so intricate that they could easily exhibit weakly density-dependent behavior at low prey densities. Second, even if the laws governing individuals are linear, weakly density-dependent effects can appear in the coarse-graining that is necessary to formulate equations on the population level. Consider for instance the effect of a heterogeneous environment. If the population density is low then the surviving individuals are likely to be found in very advantageous locations where mortality is low. As the population grows more dangerous locations have to be settled with corresponding higher mortality rate. Even if the mortality rate is linear in every location averaging over the whole settled space can yields a more complex scaling law potentially characterized by fractional scaling exponents.

In summary we conclude that weakly density-dependent processes could exist in nature. While no direct empirical evidence for such processes is presently 
available, the corresponding invisible niches may be evident indirectly. The basic results which we have presented here can form a basis for future investigations which consider the effect of weakly density-dependent effects in the context of evolutionary or assembly models and thereby establish a direct connection to island biogeography.

\section{Acknowledgements}

This work was initiated at the Computational and Mathematical Population Dynamics Conference in Trento, Italy. The authors would like acknowledge constructive comments from two anonymous referees which considerably improved the discussion. TG would like to thank Bernd Blasius for support and discussions. AME acknowledges financial support from the Sloan Census of Marine Life FMAP (Future of Marine Animal Populations) project.

\section{References}

Abrams, P. A., 2004. When does periodic variation in resource growth allow robust coexistence of competing consumer species? Ecology 85, 372-382.

Armstrong, R. A., McGehee, R., 1976a. Coexistence of species competing for shared resources. Theor. Pop. Biol. 9 (3), 317-328, doi:10.1016/00405809(76)90051-4.

Armstrong, R. A., McGehee, R., 1976b. Coexistence of two competitors on one resource. J. Theor. Biol. 56 (2), 499-502. 
Armstrong, R. A., McGehee, R., 1980. Competetive exclusion. Am. Nat. 115 (2), 151-170.

Bak, P., 1996. How Nature works: The science of self-organized criticality. Springer Verlag, Berlin.

Bracco, A., Provenzale, A., Scheuring, I., 2000. Mesoscale vortices and the paradox of the plankton. Proc. Roy. Soc. Lond. B 267, 1795-1800.

Caswell, H., Neubert, M. G., 1998. Chaos and closure terms in plankton food chain models. J. Plankton Res. 20 (9), 1837-1845.

Chase, J., Abrams, P., Grover, J., Diehl, S., Chesson, P., Holt, R., Richards, S., Nisbet, R., Case, T. J., 2002. The effects of predators on competition between their prey. Ecology Letters 5, 302-313.

Chesson, P., 2000. Mechanisms of maintenance of species diversity. Ann. Rev. Ecol. Sys. 31, 343-366, doi:0066-4162/00/1120-0343.

Ebenhöh, W., 1994. Competition and coexistence: modelling approaches. Ecol. Mod. 75-76, 83-98, doi:10.1016/0304-3800(94)90009-4.

Edwards, A. M., Bees, M. A., 2001. Generic dynamics of a simple plankton population model with a non-integer exponent of closure. Chaos, Solitons and Fractals 12, 289-300.

Edwards, A. M., Yool, A., 2000. The role of higher predation in plankton population models. J. Plankton Res. 22 (6), 1085-1112.

Gause, G. F., 1934. The struggle for existence. Williams and Wilkens, Baltimore, reprinted 1971 by Dover, New York.

Grinnell, J., 1904. The origin and the distribution of the chestnut-backed 
chickadee. Auk 21, 364-383.

Grinnell, J., 1917. The niche-relationships of the california thrasher. Auk 34, $427-434$.

Gross, T., 2004. Population Dynamics: General results from local analysis. Der Andere Verlag, Tönning.

Gross, T., 2007. Biond group homepage, http://www.pks.mpg.de/g̃ross.

Haldane, J. B. S., 1924. A mathematical theory of natural and artificial selection, Part I. Transactions of the Cambridge Philosophical Society 23, $19-41$.

Hardin, G., 1960. The competitive exclusion principle. Science 131, 1292-7.

Hastings, A., Godfray, H. C. J., 1999. Learning, host fidelity, and the stability of host-parasitoid communities. Am. Nat. 153, 295-301.

Holt, R. D., Lawton, J. H., 1994. The ecological consequences of shared natural enemies. Ann. Rev. Ecol. Sys. 25, 495-520.

Huisman, J., Weissing, F. J., 1999. Biodiversity of plankton by species oscillations and chaos. Nature 402, 407-410.

Hutchinson, G. E., 1947. A note on the theory of competition between two social species. Ecology 28, 319-321.

Hutchinson, G. E., 1959. Homage to santa rosalia or why are there so many kinds of animals? Am. Nat. 93, 145-159.

Hutchinson, G. E., 1961. The paradox of plankton. Am. Nat. 95 (882), 137145.

Kingsland, S. E., 1988. Modeling nature-episodes from the history of popula- 
tion ecology, 2nd Edition. Univ. of Chicago Press, Chicago and London.

Koch, A. L., 1974a. Coexistence resulting from an alternation of density dependent and density independent growth. J. Theor. Biol. 44 (2), 373-386, doi:10.1016/0022-5193(74)90168-4.

Koch, A. L., 1974b. Competitive coexistence of two predators utilizing the same prey under constant environmental conditions. J. Theor. Biol. 44 (2), 387-395, doi:10.1016/0022-5193(74)90169-6.

Lack, D. L., 1947. Darwin's Finches. Cambridge University Press, Cambridge.

Levin, S. A., 1970. Community equilibria and stability, an extension of the competitive exclusion principle. Am. Nat. 104 (939), 413-423.

MacArthur, R. H., 1958. Population ecology of some warblers of the northeastern coniferous forests. Ecology 39, 599-619.

Mandelbrot, B., 1967. How long is the coast of Britain - statistical selfsimilarity and fractal dimensions. Science 156 (3775), 636.

McArthur, R. H., Levins, R., 1964. Competition, habitat selection and character displacement in a patchy environment. PNAS 51, 1207-12010.

McGehee, R., Armstrong, R. A., 1977. Some mathematical problems concerning the ecological principle of competetive exclusion. J. Diff. Eqs. 23, 30-52.

Nägeli, C., 1874. The displacement of plant forms by their competitors. Sitzungsberichte der Bayerischen Akademie der Wissenschaften Mnchen 11, reviewed in J. L. Harper: A centenary in population biology, Nature 252, 526-527 (1974) doi:10.1038/252526a0.

Odum, E. P., 1961. Fundamentals of ecology. Saunders, Philadelphia, NJ. 
Rescigno, A., Richardson, I. W., 1965. On the competitive exclusion principle. Bull. Math. Biophys. 27, 85-89.

Roy, M., Pascual, M., Levin, S. A., 2004. Competitive coexistence in a dynamic landscape. Theor. Pop. Biol. 66 (4), 341-53, doi:10.1016/j.tpb.2004.06.012.

Slobodkin, L. B., 1961. Growth and regulation of animal populations. Holt, Rinehart \& Winston, New York.

Solé, R. V., Bascompte, J., Valls, J., 1992. Stability and complexity of spatially extended two-species competition. J. Theor. Biol. 159, 469-480.

Steele, J. H., Henderson, E. W., 1992. The role of predation in plankton models. J. Plankton Res. 14 (1), 157-172.

Vandermeer, J., Maruca, S., 1998. Indirect effects with a keystone predator: coexistence and chaos. Theor. Pop. Biol. 54, 38-43.

Volterra, V., 1926. Variazione e fluttuazione del numero d'individui in specie animali conviventi. Mem. Accad. Nationale Lincei 2, 31-113.

Wilson, K. G., 1975. The renormalization group: critical phenomena and the kondo problem. Rev. Mod. Physics 47 (4), 773-840.

Wilson, W. G., Abrams, P. A., 2005. Coexistence of cycling consumer species having localized interactions; armstrong and mcgehee in space. Am. Nat. $165,193-205$. 


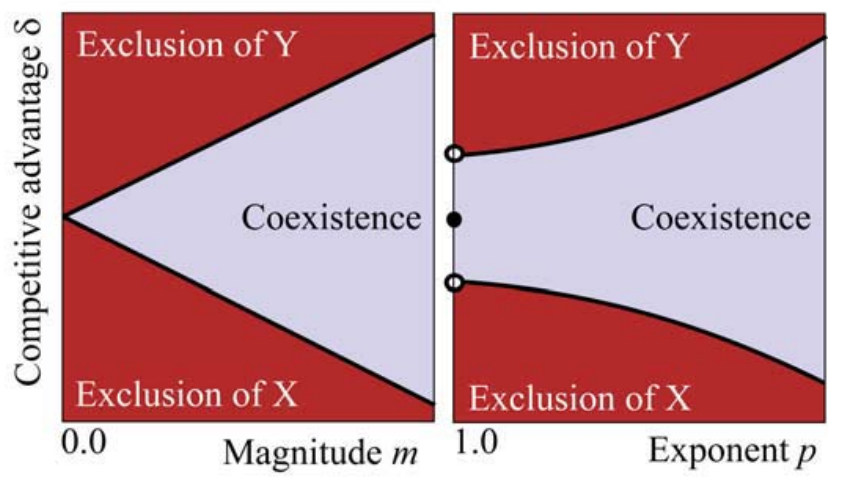

Fig. 1. Sketch of weak density-dependent mortality in comparison to weakly density-dependent mortality. Left: Weak density-dependent mortality. As the magnitude $m$ of the nonlinear mortality term is decreased the range of the competitive advantage of $\mathrm{X}, \delta=d_{\mathrm{y}}-d_{\mathrm{x}}$, in which coexistence is possible, shrinks. In the limit $m \rightarrow 0$ coexistence in only possible at one specific value of $\delta=0$. Right: Weakly density-dependent mortality. Decreasing the exponent of the nonlinear mortality term likewise reduces the range of coexistence. However, even in the limit $p \rightarrow 1$ coexistence is possible in a finite interval.Only at $p=1$ the range of coexistence shrinks to a single point in a discontinuous transition.

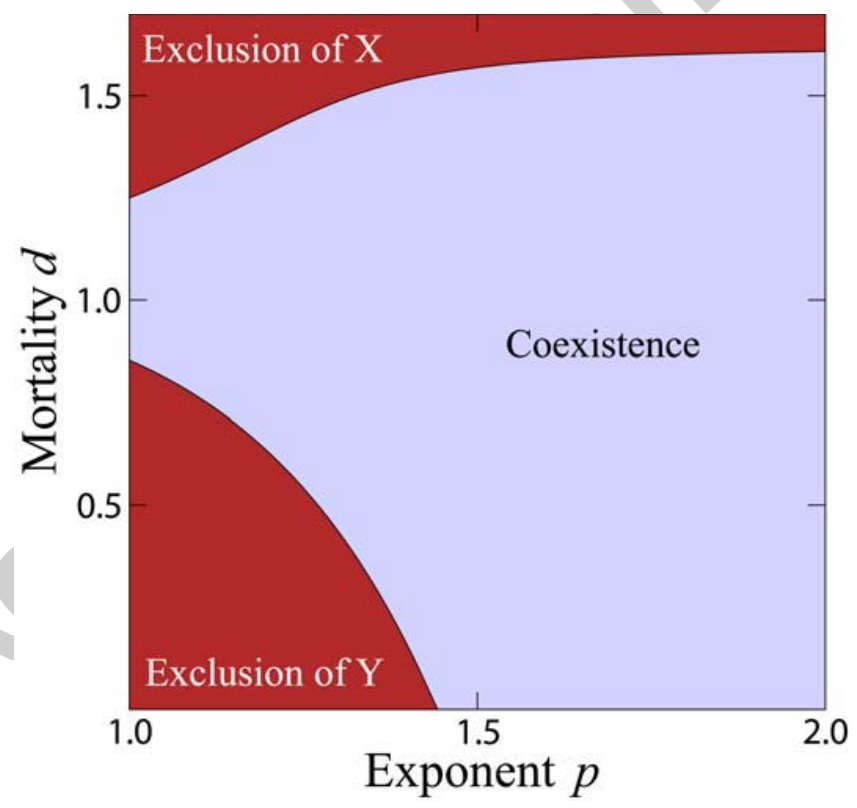

Fig. 2. Exploitative competition between two species $\mathrm{X}, \mathrm{Y}$ in a specific example. The regions of coexistence (light blue) and exclusion (dark red) are shown depending on the density-independent mortality $d$ of species $\mathrm{X}$ and the exponent $p$ of the density-dependent mortality term of both species. Even for $p \rightarrow 1$ coexistence is mathematically possible in a finite range. 


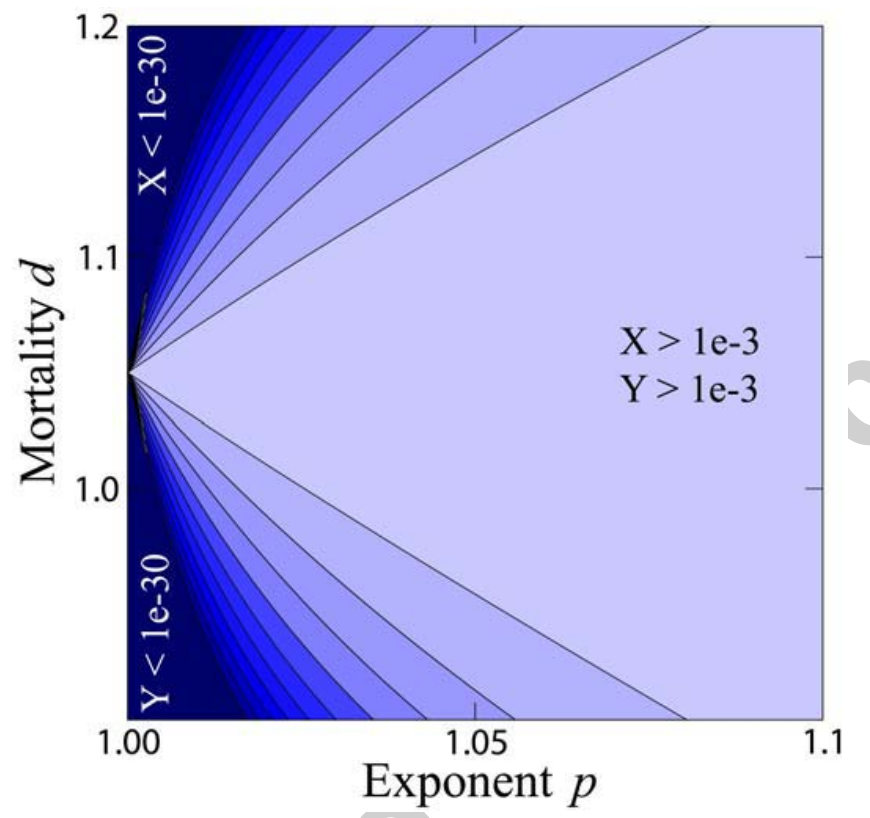

Fig. 3. Density of the weaker competitor in the competition between two species $\mathrm{X}, \mathrm{Y}$. Coexistence is mathematically possible in the whole parameter space shown (see Fig. 2). In the dark region on the left the density of the weaker competitor is below $10^{-30}$. Every line marks an increase by a factor of $10^{3}$ up to a density $10^{-3}$. In the case of weakly nonlinear mortality ( $\operatorname{small} p$ ) the range of the parameter $d$ for which the weaker competitor can maintain high densities (e.g. light blue region) becomes small. However, in a system of sufficient size coexistence at lower densities is still possible in a wide range of $d$. 


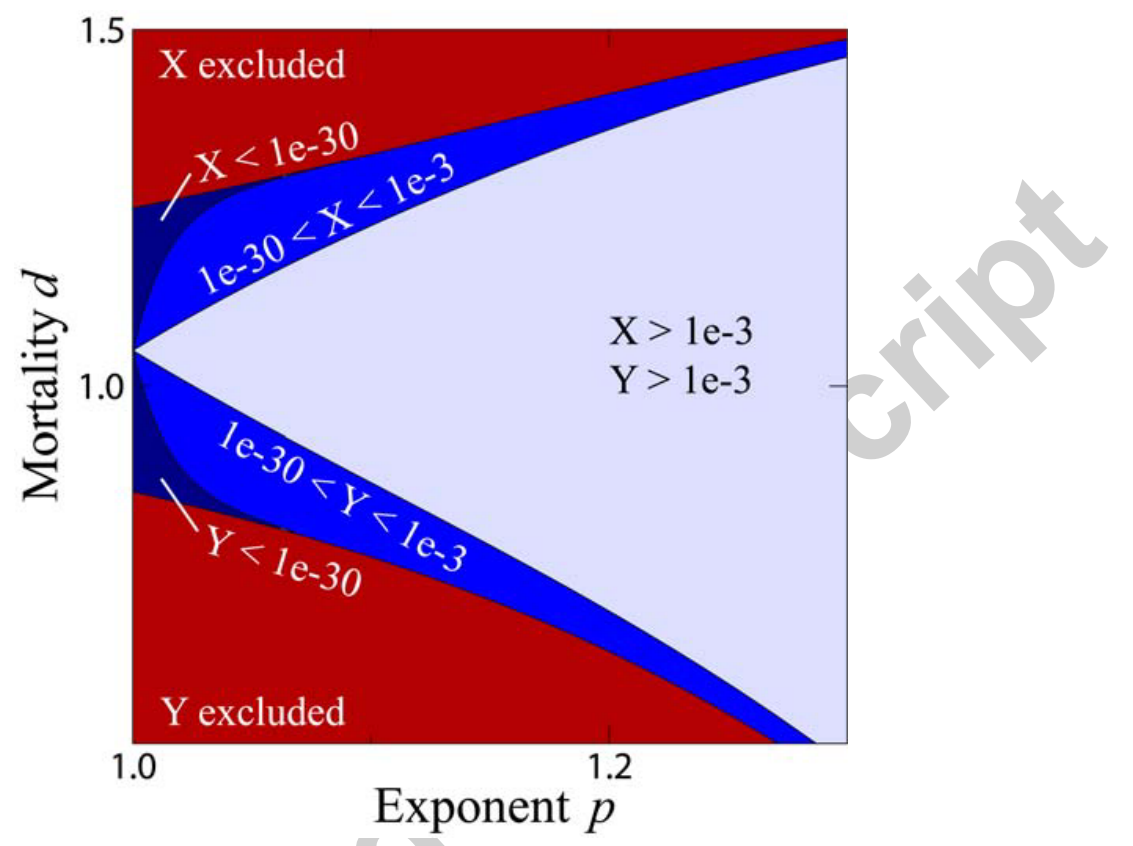

Fig. 4. Effect of different cutoff densities on coexistence between two species. Species coexistence is theoretically possible in the blue regions and impossible in the red region. The shade of the blue indicates the density of the weaker competitor. Since small densities effectively prohibit survival in small systems the effective coexistence region depends on the size of the system. This size dependence is not of importance in systems with density-independent $(p=1)$ or strongly density-dependent (say, $p>1.2$ ) mortality, but is has a strong impact in systems with weakly density-dependent mortality, e.g., $p=1.05$. 